\title{
Short term outcome of arthroscopic drilling of osteochondral lesions of the talus
}

\author{
G.A.Hosny ${ }^{1}$, H.M.El-Mowafi ${ }^{2}$, A.S.Rizk ${ }^{1}$, M.S.Abouzied ${ }^{1}$ and M.A.Mourad $^{1}$ \\ ${ }^{1}$ Orthopaedic surgery, Dept., Faculty of Medicine, Benha Univ., Benha, Egypt \\ ${ }^{2}$ Orthopaedic surgery, Dept., Faculty of Medicine, Mansoura Univ., Mansoura, Egypt \\ E-mail: dr.mahady1985@gmail.com
}

\begin{abstract}
Background: Osteochondral lesions of the talus (OLT) are any defects involving either the articular surface or the subchondral bone of the talus that encompass osteochondritis dissecans, osteochondral defects, and osteochondral fractures. These lesions often arise idiopathically or are related to acute trauma or repetitive microtrauma. The arthroscopic technique allows precise access to the site of injury and less tissue damage, allowing faster and functional recovery with fewer complication inherent to medial malleolus osteotomy or miniarthrotomy such as Infection, neurovascular injury, risk of injury to surrounding structures, risk of consolidation, delay or pseudoarthrosis and joint degeneration in the long term and increasing the possibility of the osteoarthritis. The main goal of this study was to evaluate the short term results of arthroscopic drilling of osteochondral lesions of the talus including preoperative assessment, operative techniques and short term result. Methods: The study was conducted at orthopedic surgery department- Behna university hospital on 20 patients with osteochondral lesion talus who arrived to the outpatient clinics of orthopedic surgery department- in the period between (June 2019 to September 2020) and are fulfilling the inclusion and exclusion criteria. Results: Three-quarters of the patients (75.0\%) showed the traumatic mechanism of injury. The mean weight was $64 \mathrm{~kg}$. About two-thirds (65.05) play sports. Only $10.0 \%$ of the patients had DM. Most patients were re-creational atheletes $(65.0 \%)$. Most patients showed chondral lesion type (90.0\%). The mean size was $1.1 \mathrm{~cm}$. The most frequent location was posteromedial $(55.0 \%)$, followed by anterolateral $(35.0 \%)$ and central $(10.0 \%)$. The most frequent radiographic classification was IIa $(40.0 \%)$, followed by III $(25.0 \%)$, while the least frequent classification was IV $(15 \%)$.The most frequent MRI staging was IIa (40.0\%), while the least frequent was V (5.0\%). The most frequent CT staging was IIa (30.0\%), while the least frequent were I, IIb, and IV (15\% for each). The most frequent arthroscopic staging was B (30.0\%), while the least frequent was A (5.0\%). Conclusion: Treatment of osteochondral injuries of the talus by arthroscopy and injury resection with drilling and debridement improves pain and function of the ankle, regardless of the stage of the injury and its medial or lateral location.
\end{abstract}

Key words: Arthroscopic Drilling, Osteochondral Lesions, The Talus.

\section{Introduction}

Osteochondral lesions of the talus (OLT) are any defects involving either the articular surface or the subchondral bone of the talus that include osteochondritis dissecans, osteochondral defects, and osteochondral fractures [1].

These lesions often arise idiopathically or are related to acute trauma or repetitive microtrauma. OLTs are challenging to treat because of their complex geometry and poor innate biology. Nonoperative treatment consisting of physical therapy, nonsteroidal anti-inflammatory medications, and protected weight bearing may be successful in treating approximately $50 \%$ of cases [2].

Several treatment strategies exist to address OLTs, falling into the general categories of cartilage repair, cartilage regeneration, or cartilage replacement. Cartilage repair strategies include bone marrow stimulation with microfracture and drilling. Cartilage regeneration strategies include autologous chondrocyte implantation and its derivatives, along with autologous matrix-induced chondrogenesis from bone marrow derived cells or platelet-rich plasma. Cartilage replacement strategies include osteochondral autografting or allografting and particulated juvenile cartilage allograft transplantation [3].

Microfracture has long been the gold standard for small-scale cartilage repair in the ankle because of its ease of performance and low cost [3]. However, microfracture generates biomechanically and biologically inferior fibrocartilage [4]. Despite this, microfracture for OLTs has shown acceptable symptomatic and functional improvement, at least in the short term [5].

Arthroscopic debridement and bone marrow stimulation (i.e., drilling or microfracturing) is considered the primary surgical treatment of osteochondral defects of the talus. Advantages of this procedure are the relative simplicity of the technique, outpatient treatment, low costs, and early rehabilitation. Depending on its location, the osteochondral defect can be accessed through an anterior or posterior arthroscopic approach. [6]

The aim of this work is to evaluate the short term results of arthroscopic drilling of osteochondral lesions of the talus including preoperative assessment, operative techniques and short term result.

\section{Patients and Methods \\ Technical design: \\ - Type of the study: Case serious study. \\ - Study setting: The study was conducted at orthopedic surgery department- Behna university hospital \\ Study patients}

The target population for this study were 20 patients with osteochondral lesion talus who arrived to the outpatient clinics of orthopedic surgery department- 
Benha university hospital in the period between (June 2019 to September 2020) and are fulfilling the inclusion and exclusion criteria.

\section{Inclusion Criteria:}

- This study will include Patient with one of the following criteria:

- Osteochondral lesion talar dome (anterior posterior or medial - lateral)

- No exclusion regarding age, sex.

- Size of the lesions smaller than $15 \mathrm{~mm}$.

Exclusion Criteria:

Any cases with the following criteria will be excluded

- Lesions larger than $15 \mathrm{~mm}$.

- Ankle osteoarthritis.

- Concomitant ankle pathology (tibial OCD, instability, tendonitis)

- Advanced osteoporosis.

- Infectious pathology.

- Malignancy

- Ankle malalignment.

Demographic features

Sex incidence:

There were 18 males and 2 females.

Age incidence $\pm 10 \mathrm{y}$.

The mean age of the studied patients was 31 years

\section{Mechanism of injury}

Out of the 20 patients, there were 15 the patients showed the traumatic mechanism of injury and 5 patients showed non-traumatic mechanism of injury.

\section{Lesion characteristics}

18 patients showed chondral lesion type patients and 2 patient showed sub chondral lesion type. The most frequent location was posteromedial in 11 patients, followed by anterolateral was in 7 patients and central medial was in 2 patients

\section{Patient evaluation}

Patient history:

- Clinical history was taken from the patient in the sort of name, sex, age, job, address and smoking habits.

- Associated illness like diabetes, hypertension and cardiac condition.

- Patients were asked about the mechanism of injury and if there are any associated injuries.

\section{Clinical examination}

Standard Ankle examination was performed in the form of:

- Weight of the patient.

- Tenderness \& Swelling.

- Compare the affected ankle to the contralateral Side.

- ROM of both the tibiotalar joint and the subtalar joint.

- palpating the medial and lateral aspects of the talar dome

- Skin condition.

- Neurovascular examination

- Ankle instability
- Ankle deformity

Also examination of associated injuries was performed.

Radiological evaluation:

- Radiological examination included plain radiographs (x-rays standing anteroposterior, mortise and lateral) of both ankles.

- For further diagnostic evaluation MRI or computed tomography of the affected ankle which made with the ankle in maximum planter flexion in order to be able to both determines the size, location, shape.

Classification of the fracture

Radiographic classification by Berndt and Harty ${ }^{(7)}$ :

- Stage I: Small area of subchondral compression

- Stage II: Partial fragment detachment.

- Stage III: Complete fragment detachment but not displaced

- Stage IV: Avulsed fragment into joint.

CT classification by Ferkel et al ${ }^{(8)}$ :

- Stage I: subchondral cyst but intact joint surface;

- Stage IIa: subchondral cyst with open cartilage;

- Stage IIb: open cartilage, nondisplaced fragment;

- Stage III: subchondral cyst, nondisplaced fragment;

- Stage IV: displaced fragment.

MRI classification by Hepple and et al ${ }^{(9)}$ :

- Stage I: Articular cartilage edema

- Stage IIa: Cartilage injury with underlying fracture and surrounding bony edema

- Stage IIb: Stage 2a without surrounding bone edema

- Stage III: Detached but nondisplaced fragment

- Stage IV: Displaced fragment

- Stage V: Subchondral cyst formation.

Arthroscopic classification by Ferkel ${ }^{(10)}$ :

- Stage A: soft, smooth cartilage;

- Stage B: rough cartilage;

- Stage C: fibrillation and fissures;

- Stage D: flap or naked bone;

- Stage E: sequestrum in place;

- Stage F: displaced fragment.

Surgical Technique

Preoperative assessment

- Preoperative Labs: CBC, INR, SGOT, SGPT, Urea, Creatinine.

- Preoperative Imaging: included anteroposterior, mortise and lateral radiographs, for further diagnostic evaluation MRI or computed tomography of the affected ankle.

- The preoperative shape of OLT classified according to the modified Berndt and Harty's staging system.

- Preoperative assessment of VAS score and AOFAS score.

Surgical procedure

Consent

- Informed consent was taken from the patients.

Anesthesia

- All the patients were anaesthetized by Spinal anesthesia. 


\section{Position}

Patients were operated on a standard radiolucent orthopedic table, in supine position. With slight elevation of the ipsilateral buttock. The heel of the affected foot rested on the very end of the operating table and applied a non-invasive ankle distractor.

The preferred approach of most lesions is anterior arthroscopy through an anteromedial and an anterolateral portal with the ankle in full planter flexion. With a probe, the location of OLT was identified, Debridement and bone marrow stimulation (micro fracturing, drilling), the object is to partially destroy the calcified zone that is often present and to create openings into the subchondral bone.

Postoperative care:

Active planter flexion and dorsiflexion are encouraged. Partial weight bearing is allowed as tolerated to full weight bearing within 2-4 weeks in patients with small lesions up to one $\mathrm{cm}$, while large lesions require partial weight bearing up to 6 weeks. Full return to normal activities is allowed 3 months after surgery.

The post- operative follows up includes both clinical evaluation using AOFAS scoring system and VAS score for pain, routine weight bearing radiographs (anteroposterior and lateral views) at three months and one-year post-operative.
Method of Assessment and outcome evaluation:

The patient data will include age, gender, weight, injury mechanism, type, size and location of lesions, duration of surgery, duration of admission, and time from surgery to full return to normal and sporting activities.

We used the visual analog scale (VAS) is a pain rating scale first used by Hayes and Patterson in 1921. Scores are based on self-reported measures of symptoms that are recorded with a single handwritten mark placed at one point along the length of a $10-\mathrm{cm}$ line that represents a continuum between the two ends of the scale - "no pain" on the left end $(0 \mathrm{~cm})$ of the scale and the "worst pain" on the right end of the scale $(10 \mathrm{~cm})$. Measurements from the starting point (left end) of the scale to the patients' marks are recorded in centimeters and are interpreted as their pain. ${ }^{(11)}$

\section{Results}

Lesion characteristics in the studied patients

Most patients showed chondral lesion type $(90.0 \%)$. The mean size was $1.1 \mathrm{~cm}$. The most frequent location was posteromedial $(55.0 \%)$, followed by anterolateral (35.0\%) and central medial (10.0\%). (Table $1 \&$ figure 1 )

Table 1: Lesion characteristics in the studied patients

\begin{tabular}{llcc}
\hline \multicolumn{3}{c}{ Lesion characteristics } \\
\hline Type & Chondral & $\mathbf{n}(\%)$ & $18(90.0)$ \\
& Subchondral & $\mathbf{n}(\%)$ & $2(10.0)$ \\
Size $(\mathbf{c m})$ & Mean \pm SD & & $1.1 \pm 0.2$ \\
Location & Anterolateral & $\mathbf{n}(\%)$ & $7(35.0)$ \\
& Central & $\mathbf{n}(\%)$ & $2(10.0)$ \\
& Posteromedial & $\mathbf{n}(\%)$ & $11(55.0)$ \\
\hline
\end{tabular}

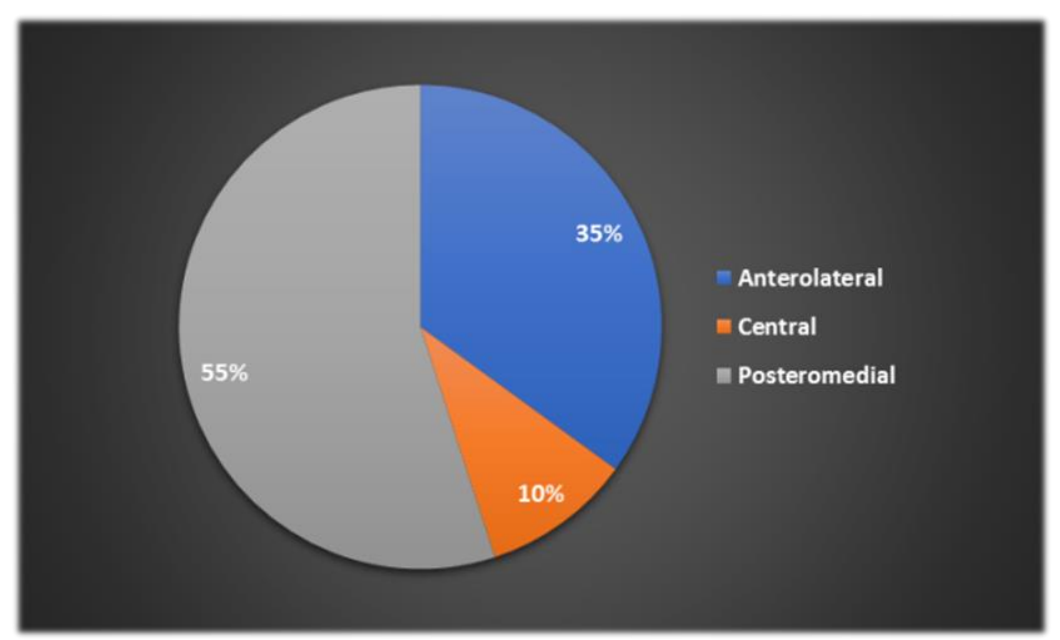

Fig. (1) Location of the Lesions in the studied patients.

\section{Radiographic classification in the studied patients}

- The most frequent radiographic classification was IIa $(40.0 \%)$, followed by III $(25.0 \%)$, while the least frequent classification was IV (15\%). (Table2) 
Table (2) Radiographic classification in the studied patients.

\section{MRI staging in the studied patients}

\begin{tabular}{llc}
\hline & & n (\%) \\
\hline Radiographic classification & IIa & $8(40.0)$ \\
& IIb & $4(20.0)$ \\
& III & $5(25.0)$ \\
& IV & $3(15.0)$ \\
\hline
\end{tabular}

- The most frequent MRI staging was IIa (40.0\%), while the least frequent was V (5.0\%). (Table 3)

Table (3) MRI staging in the studied patients

\begin{tabular}{llc}
\hline & & $\mathbf{n}(\boldsymbol{\%})$ \\
\hline MRI staging & IIa & $8(40)$ \\
& IIb & $3(15.0)$ \\
& III & $5(25.0)$ \\
& IV & $3(15.0)$ \\
& V & $1(5.0)$ \\
\hline
\end{tabular}

CT staging in the studied patients

- The most frequent CT staging was IIa (30.0\%), while the least frequent were I, IIb, and IV (15\% for each) (Table 4).

Table (4) CT staging in the studied patients.

\begin{tabular}{llc}
\hline & & n (\%) \\
\hline CT staging & I & $3(15.0)$ \\
& IIa & $6(30.0)$ \\
& IIb & $3(15.0)$ \\
& III & $5(25.0)$ \\
& IV & $3(15.0)$ \\
\hline
\end{tabular}

Arthroscopic staging in the studied patients

- The most frequent arthroscopic staging was B (30.0\%), while the least frequent was A (5.0\%). (Table 5)

Table (5) Arthroscopic staging in the studied patients.

\begin{tabular}{llc}
\hline & & n (\%) \\
\hline Arthroscopic staging & A & $1(5.0)$ \\
& B & $6(30.0)$ \\
& C & $2(10.0)$ \\
& D & $3(15.0)$ \\
& E & $5(25.0)$ \\
& F & $3(15.0)$ \\
\hline
\end{tabular}

AOFAS score in the studied patients pre and post-surgery

- AOFAS score showed an overall significant difference between different times (P-value $<0.001)$. Post hoc showed that it was significantly higher at 1 year (93.3) compared to before surgery (70) and at 3 months (84.0). Also, it was significantly higher at 3 months (84) compared to before surgery (70). Table (6) figure (2)

Table (6) AOFAS score in the studied patients pre and post-surgery.

\begin{tabular}{lll}
\hline AOFAS score & Mean \pm SD & P-value \\
\hline Before surgery & $70 \pm 4^{\mathrm{a}}$ & $<0.001$ \\
At 3 months & $84 \pm 2^{\mathrm{b}}$ & \\
At 1 year & $93.3 \pm 3.1^{\mathrm{c}}$ & \\
\hline
\end{tabular}

Repeated measures ANOVA was used. Post hoc was done using Bonferroni method. Different letters indicate significant pair 


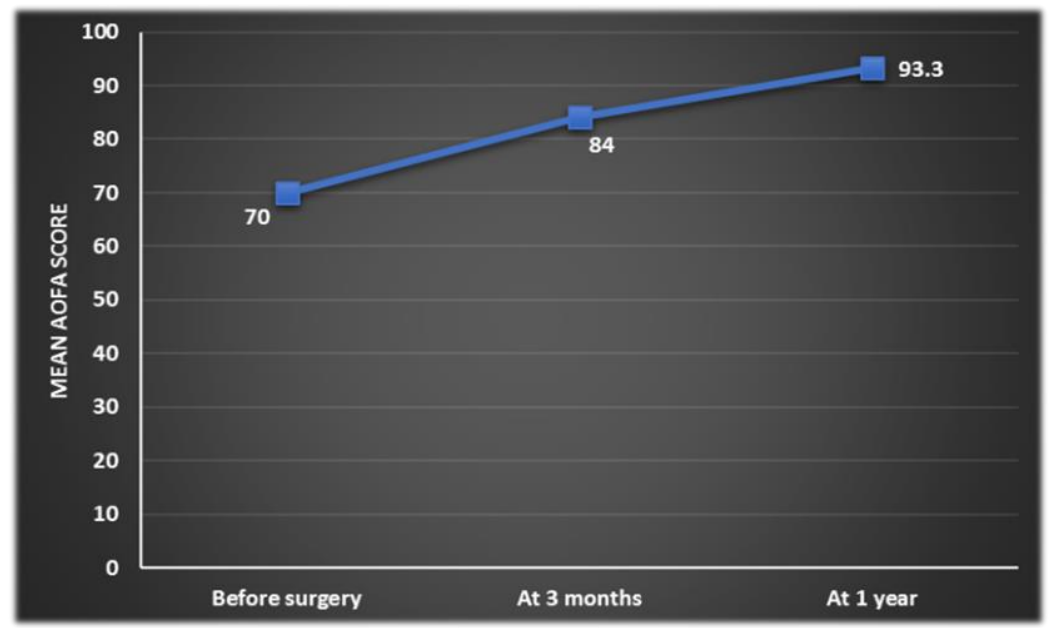

Fig. (2) AOFAS score in the studied patients pre and post-surgery.

\section{VAS score in the studied patients pre and post-surgery}

- The median VAS score showed an overall significant difference between different times (P-value $<0.001)$. Post hoc showed that it was significantly lower at 1 year (0) compared to before surgery (5) and at 3 months (2). Also, it was significantly lower at 3 months (2) compared to before surgery (5) (Table7 \& figure 3 ).

Table (7) VAS score in the studied patients pre and post-surgery.

\begin{tabular}{lcc}
\hline VAS score & Median (range) & P-value \\
\hline Before surgery & $5(5-6)^{\mathrm{a}}$ & $<0.001$ \\
At 3 months & $2(2-4)^{\mathrm{b}}$ & \\
At 1 year & $0(0-2)^{\mathrm{c}}$ & \\
\hline
\end{tabular}

Friedman's test was used. Post hoc was done using Bonferroni method. Different letters indicate significant pair

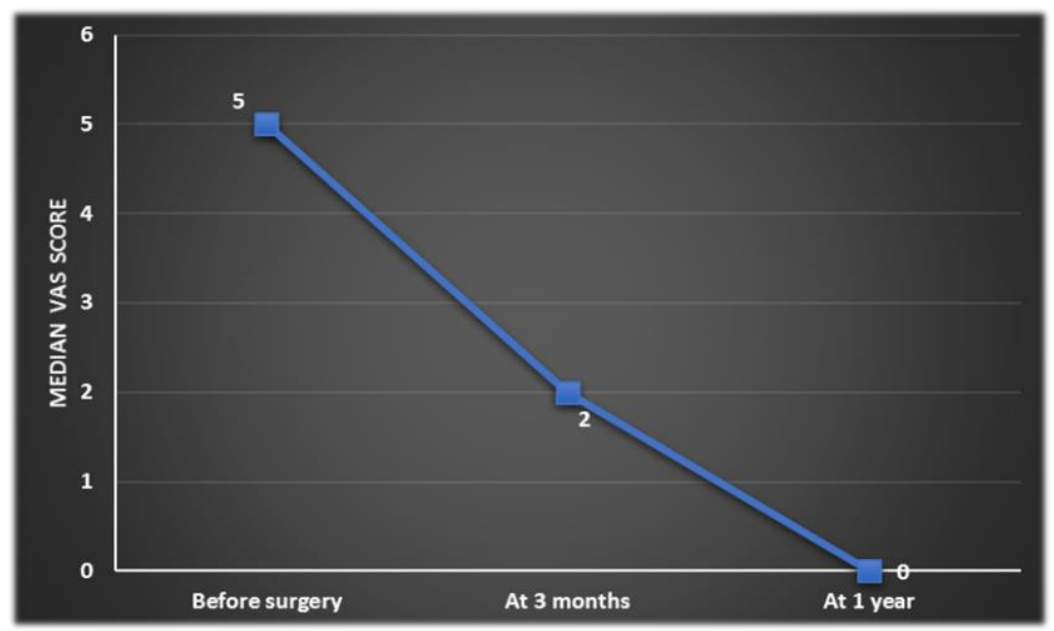

Fig. (3) VAS score in the studied patients pre and post-surgery

\section{Discussion}

In our study most patients showed chondral lesion type $(90.0 \%)$. The mean size was $1.1 \mathrm{~cm}$. The most frequent location was posteromedial $(55.0 \%)$, followed by anterolateral $(35.0 \%)$ and central medial $(10.0 \%)$. We found no difference between medial and lateral injuries in successful outcome.

In the study Araujo et al. [12] found that the improvement of pain and ankle function was significant in both the medial injuries as the lateral ones. Twenty- three $(67.6 \%)$ injuries of the 34 medial injuries were stage III or IV. Eleven injuries (52.3\%) of the 21 lateral injuries were stage III or IV. Schimmer et al. [13] found better results with medial injuries, but only $26.3 \%$ of them were stage III or IV. Guo et al. [14] found no differences between medial or lateral injuries, and all lateral injuries and $86 \%$ medial injuries were stage III or IV. 
In our study the most frequent radiographic classification was IIa $(40.0 \%)$, followed by III $(25.0 \%)$, while the least frequent classification was IV (15\%).

In the study Araujo et al. [12] the injuries were classified radiologically according to Berndt and Harty and their location as anterolateral, anteromedial, posteromedial and posterolateral. Among the 34 medial injuries, $11(32.3 \%)$ were ranked as stage II, $20(58.8 \%)$ as stage III, three $(8.8 \%)$ as stage IV. Among the 21 lateral injuries, $10(47.6 \%)$ were stage II, $10(47.6 \%)$ were stage III and one (4.8\%) stage IV. Central injuries were not found.

In our study the most frequent MRI staging was IIa $(40.0 \%)$, while the least frequent was V $(5.0 \%)$. In our study the most frequent CT staging was IIa (30.0\%), while the least frequent were I, IIb, and IV (15\% for each). In our study the most frequent arthroscopic staging was B $(30.0 \%)$, while the least frequent was A $(5.0 \%)$.

In the study Araujo et al. [12] used CT and magnetic resonance imaging to assist the classification and evaluation of the integrity of the lateral ankle ligaments.

In our study the median duration of surgery was 90 minutes and ranged from 90 to 100 minutes. The mean hospital stay was two days. The mean time until return to normal activities was 168 days.

In our study that AOFAS score showed an overall significant difference. Post hoc showed that it was significantly higher at 1 year (93.3) compared to before surgery (70) and at 3 months (84.0). Also, it was significantly higher at 3 months (84) compared to before surgery (70).

Guo et al. (14) also found significant improvement, with an average score of $90.16 \pm 9.96$ postoperatively as compared to 70.81 \pm 6.96 preoperatively by the AOFAS scale. Choi s. et al. [15] found that the AOFAS ankle-hindfoot scale showed an improvement from 71.0 points (range, 47.0-84.0) preoperatively to 89.5 points (range, 63.0-100) at the final follow-up.

In our study the median VAS score showed an overall significant difference. Post hoc showed that it was significantly lower at 1 year (0) compared to before surgery (5) and at 3 months (2). Also, it was significantly lower at 3 months (2) compared to before surgery (5).

Guo et al. [14], using a visual scale, found good correlation between AOFAS score and patient's visual scale. . Choi s. et al. [15] found that The VAS scores showed an improvement from 6.2 points (range, 4.09.0) preoperatively to 1.7 points (range, 0-6.0) at the final follow-up.

This study demonstrated a significant improvement of pain and ankle function after treatment of osteochondral injuries of the talus by arthroscopy, assessed by the AOFAS scale.

Tol et al. [16] found $88 \%$ good and excellent results in patients with stage III injuries or more advanced injuries who underwent arthroscopy under the same conditions as the present study. Chuckpaiwong et al. [17] found $100 \%$ success in injuries smaller than 1.5 $\mathrm{cm}$ diameter, although another study [12] associated better results to injuries smaller than $1 \mathrm{~cm}$. Guo et al. [12], also found significant improvement, with an average score of $90.16 \pm 9.96$ postoperatively as compared to $70.81 \pm 6.96$ preoperatively by the AOFAS scale.

The results of non-operative treatment were low compared to operative treatment. In spite of this, nonoperative treatment should always be the first treatment to be considered. Most publications on treatment of osteochondral lesions of the talus involve arthroscopic excision, curettage and bone marrow stimulation, ACI and OATS. They scored success percentages of 85,76 and 87 , respectively. ACI is a relatively expensive technique, and OATS gives morbidity from knee complaints in a relevant number of patients - up to $36 \%$. [18]

\section{Conclusion}

Treatment of osteochondral injuries of the talus by arthroscopy and debridement with drilling improves pain and function of the ankle, regardless of the stage of the injury and its medial or lateral location in conclusion, good functional outcomes and improved quality of life according to AOFAS and VAS were maintained after arthroscopic drilling of osteochondral lesions of the talus.

\section{References}

[1] CA.Looze, J.Capo, MK.Ryan. Evaluation and management of osteochondral lesions of the talus. Cartilage. Vol. 8, pp.19-30,2017.

[2] ME.Easley, LD.Latt, JR.Santangelo. Osteochondral lesions of the talus. J Am Acad Orthop Surg vol. 18: pp.616-630,2010.

[3] TJ.Dekker, PK.Dekker, DM.Tainter. Treatment of osteochondral lesions of the talus: A critical analysis review. JBJS vol.28; no.5(3): e4,2017.

[4] HY.Yang, KB.Lee. Arthroscopic microfracture for osteochondral lesions of the talus: Secondlook arthroscopic and magnetic resonance analysis of cartilage repair tissue outcomes. J Bone Joint Surg Am vol.102: pp.10-20,2020.

[5] J.Dahmen, KTA.Lambers, ML.Reilingh. No superior treatment for primary osteochondral defects of the talus. Knee Surg Sports Traumatol Arthrosc vol.26: pp.2142-2157,2018.

[6] CD.Murawski, JG.Kennedy. Operative treatment of osteochondral lesions of the talus. J Bone Joint Surg Am. Vol.95, pp.1045-54,2013.

[7] J.Christiaan, S.Laura, M.Mario. Arthroscopic treatment of osteochondral defects of the talus: outcomes at eight to twenty years of follow up. J Bone Joint Surg Am vol. 20; (6): pp.519-25. ,2013.

[8] RD.Ferkel, M.Robert, A.Gregory. Arthroscopic treatment of osteochondral lesions of the talus: 
long-term results. AM J Sport Med vol. 36, pp.1750-62,2010.

[9] Chen-Chie Wang, C.Kai, H.Ing. Current treatment concepts for osteochondral lesions of the talus. Tzu Chi Med J vol. 33, pp.243249,2020 .

[10] Seung-Won Choi, L.Gun, L.Keun-Bae. Arthrsocopic microfracture for osteochondral lesions of the talus. The American Journal of Sports Medicine vol.48, pp.153-158,2019.

[11]D.A. Delgado, B.S.Lambert, N.Boutris. Validation of Digital Visual Analog Scale Pain Scoring with a Traditional Paper-based Visual Analog Scale in Adults. J. Am. Acad. Orthop. Surg. Glob. Res. Rev vol. 2, e088,2018.

[12] Araujo, Mariana Korbage de. Arthroscopic treatment of osteochondral lesion of the talus. Acta Ortopédica Brasileira [online]., vol., 24, [Accessed 21 June 2021], pp. 32-34,2016.

[13] RC.Schimmer, W.Dick, B.Hintermann. The role of ankle arthroscopy in the treatment strategies of osteochondritis dissecans lesions of the talus. Foot Ankle Int. vol. 22, pp.895-900,2001.

[14]QW.Guo, YL.Hu, C.Jiao, CL.Yu, YF.Ao. Arthroscopic treatment for osteochondral lesions of the talus: analysis of outcome predictors. Chin Med J (Engl). vol. 123, pp.296-300,2010.

[15]SW.Choi, GW.Lee, KB.Lee. Arthroscopic Microfracture for Osteochondral Lesions of the Talus: Functional Outcomes at a Mean of 6.7 Years in 165 Consecutive Ankles. Am J Sports Med. Jan; vol. 48, pp. 153-158,2020.

[16]JL.Tol, PA.Struijs, PM.Bossuyt, RA.Verhagen, van CN.Dijk. Treatment strategies in osteochondral defects of the talar dome: a systematic review. Foot Ankle Int.; vol. 21, pp.119-26. ,2000.

[17] B.Chuckpaiwong, EM.Berkson, GH.Theodore. Microfracture for osteochondral lesions of the ankle: outcome analysis and outcome predictors of 105 cases. Arthroscopy.; vol. 24, pp.106-12 ,2008.

[18] M.Zengerink, P.A.Struijs, J.L. Tol, C.N. van Dijk. Treatment of osteochondral lesions of the talus: a systematic review. Knee surgery, sports traumatology, arthroscopy: official journal of the ESSKA, vol. 18, pp. 238-246,2010. 